\title{
Grouped Multilevel Space-Time Trellis Codes
}

\author{
Marjan Baghaie A., Student Member, IEEE, Philippa A. Martin, Senior Member, IEEE, \\ and Desmond P. Taylor, Life Fellow, IEEE
}

\begin{abstract}
Space-time trellis codes (STTCs) generally provide coding and diversity gains, but only transmit one data symbol per time slot. Using higher order modulations incurs high decoding complexity and lengthy code searches. Multi-layer schemes using multiple STTCs over subgroups of antennas provide higher throughput, but require as many receive as transmit antennas and have reduced diversity gains. Here, we develop grouped multilevel STTCs that can provide the high throughput of multi-layered schemes while realizing larger diversity gains. Any number of receive antennas can be used. An example is shown that achieves $6 \mathrm{bits} / \mathrm{sec} / \mathrm{Hz}$ using 16-QAM and 4 transmit antennas.
\end{abstract}

Index Terms-Multilevel code, space time trellis code, Rayleigh fading channel, MIMO.

\section{INTRODUCTION}

$\mathbf{S}$ PACE-TIME trellis codes (STTCs) [7], [2] can simultaneously provide coding and diversity gain, but typically transmit only one data symbol per time slot. In [9], higherrate full-diversity STTCs are derived, but no examples beyond BPSK and 2-3 transmit antennas are given ${ }^{1}$. In [8], [3] throughput is increased by splitting the transmit antennas into groups and transmitting a different STTC from each group. Each STTC spans only a subset of the antennas thus limiting diversity gain. They use successive interference cancellation and require as many receive as transmit antennas.

Multilevel coding [10] allows the construction of a high complexity coded signal constellation using simple component codes. Here we utilize multilevel coding, antenna grouping [8], [3] and STTCs to develop grouped multilevel spacetime trellis codes (GMLSTTCS), capable of simultaneously providing coding gain, diversity improvement and increased spectral efficiency. More than one data symbol per time slot is achieved by grouping antennas on some levels and using a separate STTC for each group as in [8], [3]. By retaining at least one level encoded with a STTC that spans all antennas, diversity gains can be realized compared to [8], [3] while still improving throughput. A key advantage of the GMLSTTC structure is that, by using multistage decoding, any number of receive antennas can be used. Decoding complexity remains manageable even for high order modulations.

\section{SySTEM OVERVIEW}

We consider a system with $N_{t}$ transmit and $N_{r}$ receive antennas. The $M$-QAM symbol transmitted at time $t$ by the

Manuscript received July 30, 2009. The associate editor coordinating the review of this letter and approving it for publication was Z. Yan.

The authors are with the Department of Electrical and Computer Engineering, University of Canterbury, Christchurch, New Zealand (e-mail: marjan.baghaie@ieee.org, \{p.martin, taylor\}@elec.canterbury.ac.nz).

Digital Object Identifier 10.1109/LCOMM.2010.03.091591

${ }^{1}$ In addition, no performance simulation results are given in [9] making comparison difficult. $j$ th transmit antenna is denoted $Q_{t}{ }^{j}$, for $1 \leq j \leq N_{t}$. We assume a quasi-static Rayleigh fading channel model that is constant over a frame and varies independently between frames. Each subchannel fades independently. In addition, we assume perfect channel state information (CSI) at the receiver, but none at the transmitter.

The output of the $i$ th receive antenna at time $t$ is given by

$$
r_{t}^{i}=\sum_{j=1}^{N_{t}} h_{i j} Q_{t}^{j}+n_{t}^{i}, \quad i=1, \cdots, N_{r},
$$

where $n_{t}^{i}$ is the additive white Gaussian noise associated with the $i$ th receive antenna at time $t$. The path gain of the $j$ th transmit to $i$ th receive antenna subchannel is modelled as an independent complex Gaussian random variable with zero mean and variance $1 / 2$ per dimension.

\section{A. Encoding}

The structure of the GMLSTTC system is shown in Fig. 1. It uses multilevel coding and set partitioning to partition a $M$ QAM constellation into subsets of constellation points [10]. The $M$-QAM constellation is partitioned $L$ times using $\mathcal{M}$ way partitions ${ }^{2}$, where $M=\mathcal{M}^{L}$. This is shown in Fig. 2(a) for $L=2, \mathcal{M}=4$ and 16-QAM. The level 1 code chooses one of the shaded subsets and then the level 2 code chooses the actual point within the subset transmitted. Set partitioning results in increasing Euclidean distance on each level, meaning the strongest code is required on level 1.

STTCs designed for $\mathcal{M}$-QAM [2] are used as component codes on each level ${ }^{3}$. Throughout we assume $r N_{r} \geq 4$, where $r$ is the rank of the code difference matrix [2]. This results in minimum Euclidean distance dominating performance. Thus we design codes following the trace criterion [2]. The receiver applies a modified STTC decoder in each decoder stage. The output of the $k$ th component code $\mathcal{C}(k)$, denoted $\mathbf{x}_{t}(k)=\left(x_{t}(k, 1), \ldots, x_{t}\left(k, N_{t}\right)\right)$, selects the subset of constellation points on the $k$ th partitioning level.

This mapping can be implemented using the approach of [5]. In this case the STTCs directly output sequences of $\mathcal{M}$-QAM symbols, meaning $x_{t}(k, m)=\chi_{I}+j \chi_{Q} \mid \chi_{I}, \chi_{Q}$ $\in\{1,-1\}$ assuming $\mathcal{M}=4, m=1, \cdots, N_{t}$. The actual transmitted point is collectively defined by the $\mathcal{M}$-QAM symbols from all $L$ levels. We can write the transmitted $M$ QAM point from the $j$ th transmit antenna at time $t$ in terms of the $L \mathcal{M}$-QAM symbols [5] as

$$
Q_{t}^{j}=d_{x(1)} x_{t}(1, j)+d_{x(2)} x_{t}(2, j)+\ldots+d_{x(L)} x_{t}(L, j),
$$

\footnotetext{
${ }^{2}$ Note that different values of $\mathcal{M}$ could be used on each level. Then $M$ becomes the multiple of these values for all levels.

${ }^{3}$ Any codes mapped to $\mathcal{M}$-QAM can potentially be used. For example, the super orthogonal STTCs of [4] for QPSK could be used to further improve coding gain.
} 


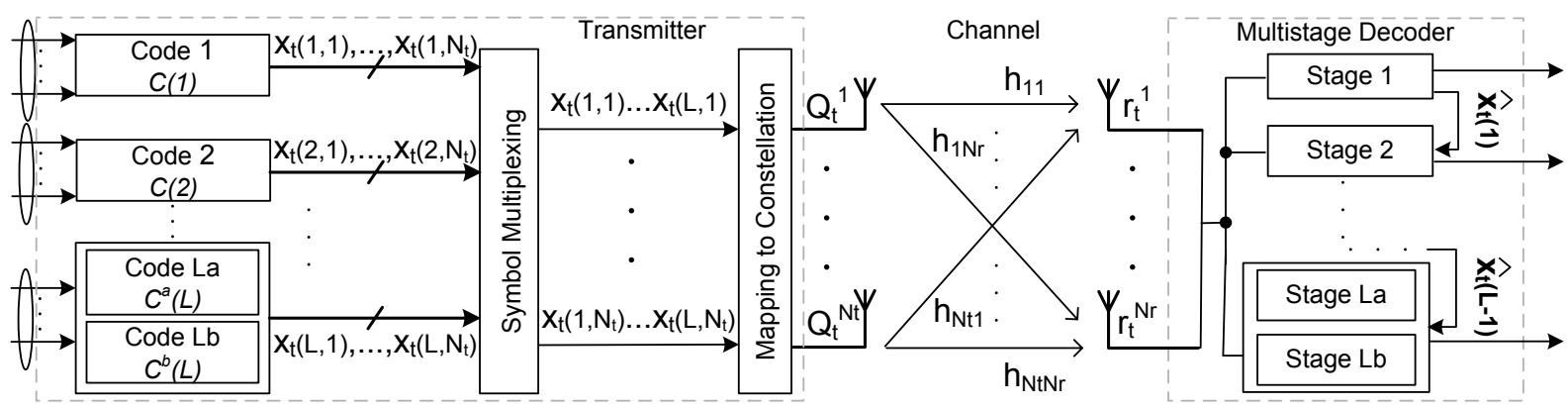

Fig. 1. Block diagram of proposed $L$-level GMLSTTC system. Level/ stage 2 to $L-1$ may use multiple codes and level $L$ can have more than 2 codes.

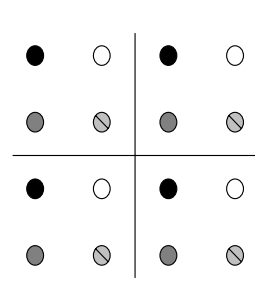

(a)

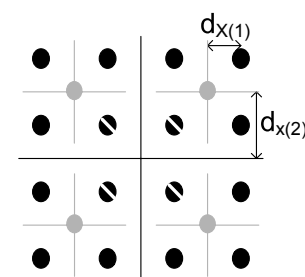

(b)
Fig. 2. Partitioning of 16-QAM using $(\mathcal{M}=4)$-way partitions and $L=2$. (a) Level 1 set partitioning into $\mathcal{M}=4$ subsets (each marked with different shaded circles). (b) The weighted sum of the level 1 (white line) and 2 (grey) 4-QAM points selects the transmitted 16-QAM point (black).

where $d_{x(1)}, \ldots, d_{x(L)}$ are the subset distances corresponding to $x_{t}(1, j), \ldots, x_{t}(L, j)$ (for all $j$ ), as shown in Fig. 2(b) for $M=16, \mathcal{M}=4$ and $L=2$. Note that the subset distances can be adjusted to provide different performance tradeoffs on each level. For equal error protection, according to the balanced distance rule [10], we want $d_{x(1)}^{2} d_{\text {free }}^{\mathcal{C}(1)}=\ldots=d_{x(L)}^{2} d_{\text {free }}^{\mathcal{C}(L)}$, where $d_{\text {free }}^{\mathcal{C}(k)}$ is the free distance of the $k$ th component code.

Level 1 always uses a single full-diversity STTC spanning all $N_{t}$ transmit antennas. However, we use multiple STTCs on some later levels in order to increase throughput over a conventional STTC. This is done by grouping antennas, as in [8], [3], on some levels. It enables the design of GMLSTTCs that transmit more than one data symbol per time slot. On level $k>1$ we split the $N_{t}$ transmit antennas into $G_{k}$ groups each of $N_{G_{k}}$ antennas. Note that each group could use a different number of antennas if desired and different antennas can be grouped on each level. A STTC for $N_{G_{k}}$ antennas and $\mathcal{M}$ QAM is used for each level $k$ group.

\section{B. Detection/Decoding}

As shown in Fig. 1, we use a multi-stage decoder with $L$ stages to decode the $L$-level GMLSTTC. The decoder starts by decoding the level 1 component code, denoted stage 1 . This code offers full-diversity over the constellation subsets it selects, allowing good diversity gains. The first level tends to dominate performance due to error propagation. Grouped levels have smaller diversity, $\min _{k=1: L}\left(N_{G_{k}}\right)$, but have better distance properties than level 1 due to set partitioning. The decision, $\widehat{\mathbf{x}}_{t}(1)$, on $\mathbf{x}_{t}(1)$ is passed to the next decoding stage and is used to decode the values of $\mathbf{x}_{t}(2)$ and so forth. The final stage of the decoder uses the decisions from levels 1 to $L-1$, namely $\widehat{\mathbf{x}}_{t}(1), \widehat{\mathbf{x}}_{t}(2), \ldots, \widehat{\mathbf{x}}_{t}(L-1)$ to obtain $\widehat{\mathbf{x}}_{t}(L)$.
Consider stage $k, k=1, \cdots, L$. Stage $k$ decodes the subset labels $\mathbf{x}_{t}(k)$ using the Viterbi algorithm to compute the path with the largest accumulated metric over the duration of a data frame. The branch metric is created using a max-log approximation $^{4}$ to the likelihood function [6]. For $1<k<L$ the outputs of the stage 1 to $k-1$ decoders (i.e. $\widehat{\mathbf{x}}_{t}(1), \ldots, \widehat{\mathbf{x}}_{t}(k-1)$ ) are available. The values of $\mathbf{x}_{t}(k+1), \ldots, \mathbf{x}_{t}(L)$ are still unknown at this stage. They are treated as "nuisance" variables and averaged out. If multiple codes are used on level $k$, then the components of $\mathbf{x}_{t}(k)$ defined by the other codes must be averaged out, while the state transition defines the remaining components.

For ease of exposition we consider a $L=2$ level GMLSTTC over $N_{t}=4$ transmit antennas. We employ a single fulldiversity STTC, $\mathcal{C}(1)$, on level 1 that spans all $N_{t}$ antennas. We use two identical STTCs on level 2 (each spanning $N_{t} / 2$ antennas), denoted $\mathcal{C}^{a}(2)$ (for antenna 1 and 2 ) and $\mathcal{C}^{b}(2)$ (for antennas 3 and 4). They produce $\mathbf{x}_{t}(2)=\left(\mathbf{x}_{t}^{a}(2), \mathbf{x}_{t}^{b}(2)\right)=$ $\left(x_{t}^{a}(2,1), x_{t}^{a}(2,2), x_{t}^{b}(2,3), x_{t}^{b}(2,4)\right)$. The decoder and metric can easily be extended to have multiple codes on any level, and to other values of $M, \mathcal{M}$ and $L$. Based on (1) and (2), the received signal at the $i$ th receive antenna at time $t$ is

$$
r_{t}^{i}=\sum_{j=1}^{N_{t}} h_{i, j}{ }^{t} d_{x(1)} x_{t}(1, j)+\sum_{j=1}^{N_{t}} h_{i, j}{ }^{t} d_{x(2)} x_{t}(2, j)+n_{t}^{i} .
$$

For a transition labelled $\mathbf{x}_{t}(1)$ we decode $\mathcal{C}(1)$ using the branch metric

$$
\begin{gathered}
\max _{\substack{\tilde{\mathbf{x}}_{t}^{a}(2) \in\left\{\mathbf{x}_{t}^{a}(2)\right\} \\
\tilde{\mathbf{x}}_{t}^{b}(2) \in\left\{\mathbf{x}_{t}^{b}(2)\right\}}} \sum_{i=1}^{N_{r}} \mid r_{t}^{i}-\sum_{j=1}^{2} h_{i, j}^{t} d_{x(2)} \tilde{x}_{t}^{a}(2, j) \\
-\sum_{j=3}^{4} h_{i, j}^{t} d_{x(2)} \tilde{x}_{t}^{b}(2, j)-\left.\sum_{j=1}^{N_{t}} h_{i, j}^{t} d_{x(1)} x_{t}(1, j)\right|^{2}
\end{gathered}
$$

Then two parallel Viterbi decoders decode $\mathcal{C}^{a}(2)$ and $\mathcal{C}^{b}(2)$. The branch metric for $\mathcal{C}^{a}(2)$ (and transition label $\mathbf{x}_{t}^{a}(2)$ ) is

$$
\begin{gathered}
\max _{\tilde{\mathbf{x}}_{t}^{b}(2) \in\left\{\mathbf{x}_{t}^{b}(2)\right\}} \sum_{i=1}^{N_{r}} \mid r_{t}^{i}-\sum_{j=1}^{2} h_{i, j}^{t} d_{x(2)} x_{t}^{a}(2, j) \\
-\sum_{j=3}^{4} h_{i, j}^{t} d_{x(2)} \tilde{x}_{t}^{b}(2, j)-\left.\sum_{j=1}^{N_{t}} h_{i, j}^{t} d_{x(1)} \widehat{x}_{t}(1, j)\right|^{2}
\end{gathered}
$$

${ }^{4}$ In simulations, this was found to incur a small (approximately constant) loss in error performance [1]. 


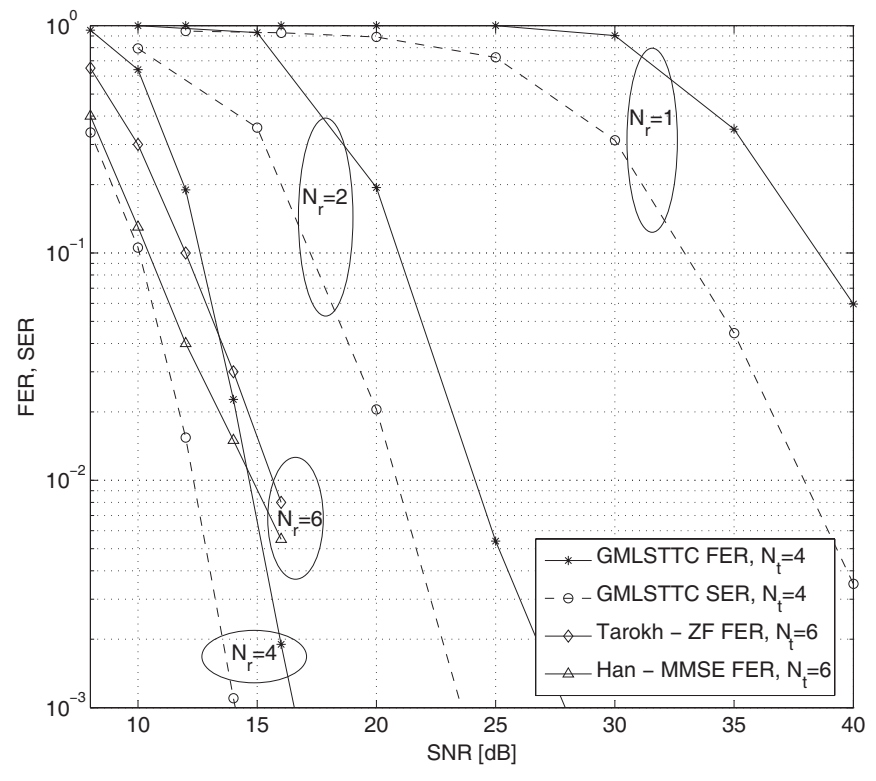

Fig. 3. FER and SER of GMLSTTC using $(M=16)$-QAM and STTCs over $(\mathcal{M}=4)$-QAM. FER of layered STTCs of [8], [3] using 4-QAM.

and for $\mathcal{C}^{b}(2)$ (and transition label $\mathbf{x}_{t}^{b}(2)$ ) is

$$
\begin{gathered}
\max _{\tilde{\mathbf{x}}_{t}^{a}(2) \in\left\{\mathbf{x}_{t}^{a}(2)\right\}} \sum_{i=1}^{N_{r}} \mid r_{t}^{i}-\sum_{j=3}^{4} h_{i, j}^{t} d_{x(2)} x_{t}^{b}(2, j) \\
-\sum_{j=1}^{2} h_{i, j}^{t} d_{x(2)} \tilde{x}_{t}^{a}(2, j)-\left.\sum_{j=1}^{N_{t}} h_{i, j}^{t} d_{x(1)} \widehat{x}_{t}(1, j)\right|^{2} .
\end{gathered}
$$

Due to the use of STTCs over $\mathcal{M}$-QAM we have $\mathcal{M}$ branches per state instead of $M=\mathcal{M}^{L}$. A single full-rank STTC would use at least $M^{N_{t}-1}$ states [7], whereas the level $k$ code in GMLSTTC uses at least $\mathcal{M}^{N_{G_{k}}-1}$ states. Complexity savings compared to a single STTC increase with constellation size. The metric calculations are more complex than for a single STTC, with level $k$ (for $G_{k}=1$ ) requiring $N_{r}\left[1+N_{t}\left(L-k+1+2^{k-1}(k-1)\right)\right]$ multiply accumulates instead of $N_{r}\left(1+N_{t}\right)$ per branch. More information can be found in [1].

\section{Simulation Results}

We present simulation results to evaluate GMLSTTC performance. We achieve $6 \mathrm{bits} / \mathrm{sec} / \mathrm{Hz}$ using $(M=16)$-QAM $\left(d_{x(2)}=2\right.$ and $\left.d_{x(1)}=1\right),(\mathcal{M}=4)$-way partitions, $L=2$ levels, $N_{t}=4$ transmit and up to $N_{r}=4$ receive antennas with a frame size of 130 symbols.

In Fig. 3 we show the frame (FER) and symbol (SER) error rates for a GMLSTTC using a 16-state STTC [2], $\mathcal{C}(1)$, designed for $N_{t}=4$ and 4-QAM on level 1 . On level 2 we use two identical component codes, $\mathcal{C}^{a}(2)$ and $\mathcal{C}^{b}(2)$, each a 4-state STTC designed for $N_{t}=2$ and 4-QAM [2]. Each STTC provides a throughput of $2 \mathrm{bits} / \mathrm{sec} / \mathrm{Hz}$, resulting in an overall throughput of $6 \mathrm{bits} / \mathrm{sec} / \mathrm{Hz}$. Results are plotted against signal to noise ratio $(S N R)$. Performance can be improved by approximately $0.75 \mathrm{~dB}$ at a FER of $10^{-3}$ (same diversity achieved) by using a 64 state code on level 1 , as this provides equal error protection for all levels.

Although not ideal, the best comparison scheme we have found for $6 \mathrm{bit} / \mathrm{sec} / \mathrm{Hz}$ is that given in [3] for $N_{t}=6$ and $N_{r}=6$. The 6 transmit antennas are divided into three layers or groups each composed of two antennas. The transmission power is equally allocated among the three layers. They use a 32-state, an 8-state and a 4-state 4-QAM STTC for $N_{t}=2$ as their component codes. The decoder starts by decoding the strongest code (32-state) and then proceeds successively to the 8-state and 4-state codes. In Fig. 3 we compare the performance of the system in [3], with our proposed 6 bits/sec/Hz GMLSTTC for $N_{t}=4$ and $N_{r}=4$ described above. The GMLSTTC has fewer states. As predicted we achieve greater diversity (as evident from the slope of the FER curves) and therefore, achieve better performance at high SNRs. A restriction of the layered system of [3], [8] is that, as with BLAST systems, it cannot work with only one receive antenna. However, GMLSTTCs can work with any number of receive antennas as shown in Fig. 3.

\section{CONCLUSIONS}

We have developed GMLSTTCs capable of simultaneously providing spectral efficiency, diversity improvement and coding gain with manageable decoding complexity. The structure is flexible and can be tuned to achieve the required balance between spectral efficiency, error performance and decoding complexity. The proposed GMLSTTC performed better than the $6 \mathrm{bits} / \mathrm{sec} / \mathrm{Hz}$ layered STTCs of [3], [8] at high SNRs due to achieving better diversity.

\section{REFERENCES}

[1] M. Baghaie A., "Multilevel space-time trellis codes for Rayleigh fading channels," ME Thesis, University of Canterbury, New Zealand, 2008, http://hdl.handle.net/10092/2101.

[2] Z. Chen, J. Yuan, and B. Vucetic, "An improved space-time trellis coded modulation scheme on slow Rayleigh fading channels," in Proc. ICC, Helsinki, Finland, pp. 1110-1116, June 2001.

[3] C. Han and D. Yuan, "An improved group detection algorithm for MLSTTC in wireless communication systems," in Proc. IEEE ICMTAS, vol. 1, pp. 1-4, Nov. 2005.

[4] H. Jafarkhani and N. Seshadri, "Super-orthogonal space-time trellis codes," IEEE Trans. Inf. Theory, vol. 49, pp. 937-950, Apr. 2003.

[5] K. Miyauchi, S. Seki, and H. Ishio, "New technique for generating and detecting multilevel signal formats," IEEE Trans. Commun., vol. 24, pp. 263-267, Feb. 1976

[6] P. Robertson, E. Villebrun, and P. Hoeher, "A comparison of optimal and sub-optimal MAP decoding algorithms operating in the log domain," in Proc. ICC, pp. 1009-1013, 1995.

[7] V. Tarokh, N. Seshadri, and A. R. Calderbank, "Space-time codes for high data rate wireless communication: performance criterion and code construction," IEEE Trans. Inf. Theory, vol. 44, pp. 744-765, Mar. 1998.

[8] V. Tarokh, A. Naguib, N. Seshadri, and A. R. Calderbank, "Combined array processing and space-time coding," IEEE Trans. Inf. Theory, vol. 45, no. 4, pp. 1121-1128, May 1999.

[9] R. Vaze and B. S. Rajan, "On space-time trellis codes achieving optimal diversity multiplexing tradeoff," IEEE Trans. Inf. Theory, vol. 52, no. 11, pp. 5060-5067, Nov. 2006.

[10] U. Waschmann, R. F. Fischer, and J. B. Huber, "Multilevel codes: theoretical concepts and practical design rules," IEEE Trans. Inf. Theory, vol. 45, pp. 1361-1391, July 1999. 